\title{
Observation of uncompensated bound charges at improper ferroelectric domain walls
}

Peggy Schoenherr, ${ }^{\dagger}$ Konstantin Shapovalov, ${ }^{\ddagger}$ ฯ Jakob Schaab, ${ }^{\dagger}$ Zewu Yan, ${ }^{\S}, \|$ Edith

D. Bourret, "Mario Hentschel, ${ }^{\perp}$ Massimiliano Stengel, ${ }^{\boldsymbol{\Phi}}, \#$ Manfred Fiebig, ${ }^{\dagger}$ Andrés Cano, ${ }^{@}$ and Dennis Meier ${ }^{*, \dagger}, \triangle$

$\dagger$ Department of Materials, ETH Zurich, Vladimir-Prelog-Weg 4, 8093 Zurich, Switzerland $\ddagger C N R S$, Université de Bordeaux, ICMCB, UPR 9048, 33600 Pessac, France 【Institut de Ciència de Materials de Barcelona (ICMAB-CSIC), Campus UAB, 08193 Bellaterra, Spain

$\S$ Department of Physics, ETH Zurich, Otto-Stern-Weg 1, 8093 Zurich, Switzerland ||Materials Science Division, Lawrence Berkeley National Laboratory, California 94720, $U S A$

$\perp 4$ th Physics Institute and Research Center SCoPE, University of Stuttgart, Pfaffenwaldring 57, 70569 Stuttgart, Germany \#ICREA-Institució Catalana de Recerca i Estudis Avançats, 08010 Barcelona, Spain @Institut Néel, CNRS \& Univ. Grenoble Alpes, 38042 Grenoble, France $\triangle$ Department of Materials Science and Engineering, Norwegian University of Science and Technology, NTNU, 7043 Trondheim, Norway

E-mail: dennis.meier@ntnu.no, andres.cano@neel.cnrs.fr

Phone: $+47(0) 73594047$

Abstract 

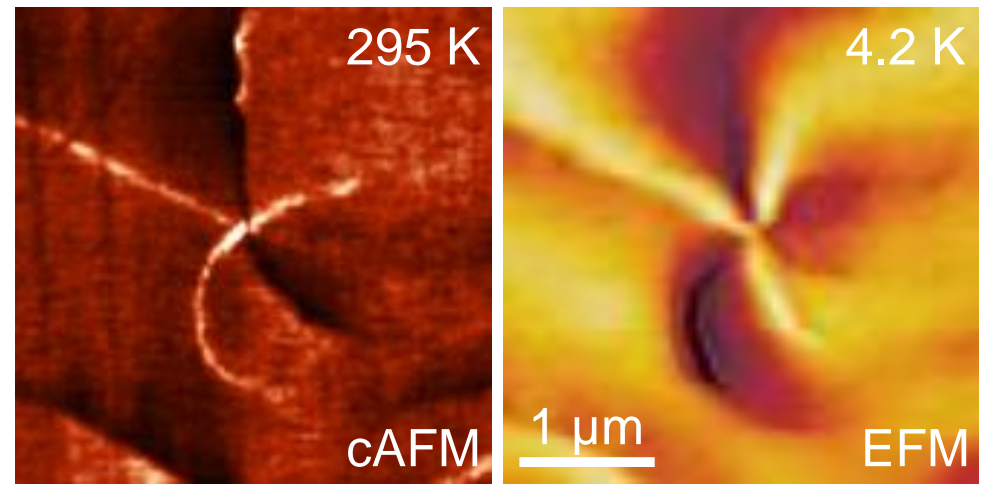

Low-temperature electrostatic force microscopy (EFM) is used to probe unconventional domain walls in the improper ferroelectric semiconductor $\mathrm{Er}_{0.99} \mathrm{Ca}_{0.01} \mathrm{MnO}_{3}$ down to cryogenic temperatures. The low-temperature EFM maps reveal pronounced electric far fields generated by partially uncompensated domain-wall bound charges. Positively and negatively charged walls display qualitatively different fields as a function of temperature, which we explain based on different screening mechanisms and the corresponding relaxation time of the mobile carriers. Our results demonstrate domain walls in improper ferroelectrics as a unique example of natural interfaces that are stable against the emergence of electrically uncompensated bound charges. The outstanding robustness of improper ferroelectric domain walls in conjunction with their electronic versatility brings us an important step closer to the development of durable and ultra-small electronic components for next-generation nanotechnology.

Keywords: Electrostatic force microscopy, domain walls, improper ferroelectric, cryogenic temperatures, hexagonal manganites

Surfaces and interfaces in complex oxide materials can be electrically polar, which leads to unusual physical behavior beyond the bulk properties. These include local electronic reconstruction phenomena such as emergent quasi-2D magnetism, multiferroicity and su- 
perconductivity. ${ }^{1}$ Recently, it has been demonstrated that polarity-driven reconstruction also occurs at ferroelectric domain walls, i.e., natural interfaces that separate regions with a different orientation of the spontaneous ferroelectric polarization. ${ }^{2-5}$ Analogous to artificially designed polar interfaces, electrons and holes redistribute in order to compensate the polarization-induced domain-wall charges, giving rise to intriguing functionalities. ${ }^{6,7}$ Domain walls in ferroelectrics develop, for example, diverse d.c. transport characteristics ranging from highly conducting to strongly insulating states, ${ }^{3,4,8-12}$ unusual a.c. conductivity, ${ }^{13,14}$ and can be used to implement elementary digital operations. ${ }^{15}$ Furthermore - in advantage to artificially designed interfaces - the domain walls can be injected, moved, and erased on demand, ${ }^{9}$ opening up completely new opportunities for interface engineering and nanoelectronics applications.

Spontaneous formation of charged domain walls is rare in proper ferroelectrics such as $\mathrm{BaTiO}_{3}$ and $\mathrm{PbZr}_{0.2} \mathrm{Ti}_{0.8} \mathrm{O}_{3}$ because of the high electrostatic costs. Using special preparation methods, ${ }^{4,16}$ charged walls can be created, but their stability critically depends on the material's ability to screen the associated diverging electrostatic potential. ${ }^{5}$

A different situation arises in so-called improper ferroelectrics; in this class of materials the polarization is a by-product that evolves as a secondary effect whilst the primary symmetry-breaking order parameter can be, for example, of structural or magnetic origin. ${ }^{17}$ As a result, charged domain walls naturally arise in the as-grown state, ${ }^{3}$ enforced by the primary order parameter, which is responsible for the symmetry breaking and domain formation. Because of the secondary nature of the polar order, charged walls are significantly more stable in improper ferroelectrics than in proper ferroelectrics and theory even predicts that they may exist without screening, representing a rare example of a stable, electrically uncompensated oxide interface..$^{5,18}$

In this article, we report on the observation of electrically uncompensated charged domain walls in hexagonal manganites. Using low-temperature scanning probe microscopy (SPM) and analytical modeling, we investigate the electronic domain-wall transport, carrier distri- 
bution and electrostatics at positively (head-to-head) and negatively (tail-to-tail) charged improper ferroelectric walls at temperatures down to $4.2 \mathrm{~K}$. By decreasing the temperature, we observe the emergence of pronounced electric far fields that originate from partially unscreened bound charges at the domain walls. The magnitude and temperature dependence of these fields are qualitatively different for head-to-head and tail-to-tail walls, which we explain based on the local distribution of screening carriers. Our low-temperature study provides new insight into the electrostatics at domain walls in improper ferroelectrics and demonstrates a new aspect of their outstanding robustness.

Ferroelectric domain walls in $\mathrm{Er}_{0.99} \mathrm{Ca}_{0.01} \mathbf{M n O}_{3}$. For our study we choose the $p$ type hexagonal semiconductor $\mathrm{Er}_{0.99} \mathrm{Ca}_{0.01} \mathrm{MnO}_{3}$. The system exhibits improper ferroelectricity below $T_{\mathrm{C}} \approx 1470 \mathrm{~K}$, with a spontaneous polarization $P_{s} \approx 6 \mu \mathrm{Ccm}^{-2}$ at roomtemperature. ${ }^{19,20}$ In the ferroelectric phase, the system develops all fundamental types of ferroelectric domain walls, namely neutral (side-by-side) as well as negatively (tail-to-tail) and positively (head-to-head) charged walls; the atomic structure and basic transport phenomena at these walls are now well-understood. ${ }^{3,21,22}$ At tail-to-tail walls, hole carriers accumulate to screen the negative bound charges, giving rise to enhanced conductance. In contrast, the screening at head-to-head walls is explained by hole depletion and the subsequent formation of an electronic inversion layer. The latter governs the conductivity at high voltage, enabling switching between insulating (low voltage) and conducting (high voltage) domain-wall behavior. ${ }^{15}$ Compared to the parent compound $\mathrm{ErMnO}_{3}$, the doping of $\mathrm{Ca}^{2+}$ enhances the bulk conductivity and, hence, improves the screening efficiency at the walls. ${ }^{23}$ Single-crystals of $\mathrm{Er}_{0.99} \mathrm{Ca}_{0.01} \mathrm{MnO}_{3}$ are grown by the pressurized floating-zone method. ${ }^{24} \mathrm{~A}$ specimen is oriented by Laue diffraction and prepared into a $70 \mu \mathrm{m}$ thick platelet with the ferroelectric polarization $(\mathbf{P} \| \mathbf{z})$ lying in the surface plane. To achieve the flat surface required for our measurements, the sample is chemo-mechanically polished using silica slurry, yielding a root-mean-square roughness of less than $1 \mathrm{~nm}$. Platinum markers with a distance of $20 \mu \mathrm{m}$ are patterned on the surface by electron beam lithography in positive tone re- 

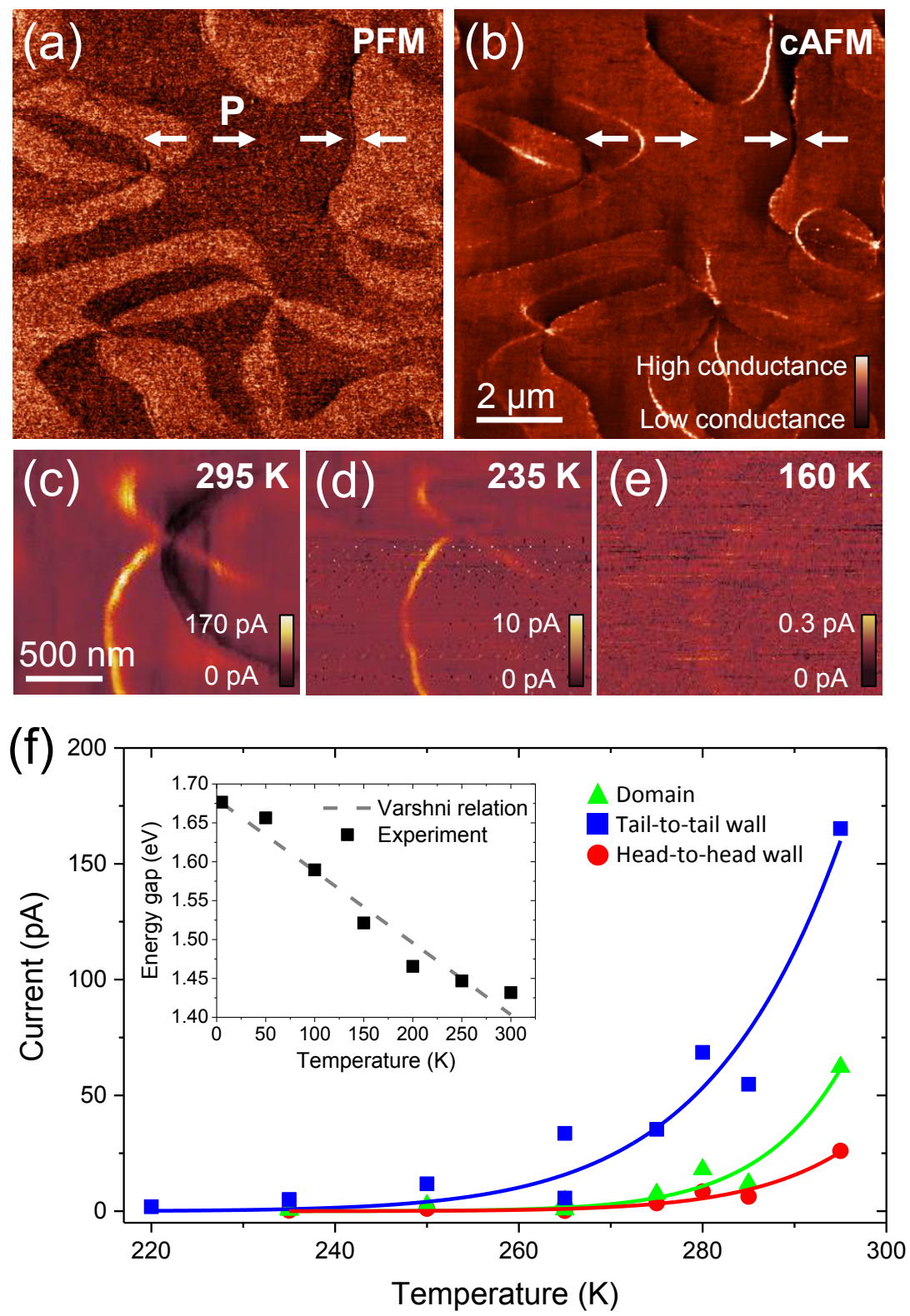

Figure 1: (a) PFM image (in-plane contrast) of the ferroelectric domain structure in $\mathrm{Er}_{0.99} \mathrm{Ca}_{0.01} \mathrm{MnO}_{3}$. The orientation of the spontaneous polarization $\mathbf{P}$ is indicated by white arrows. (b) Room-temperature cAFM image obtained in forward bias (SI Note 1) at a DC bias voltage of $3 \mathrm{~V}$ applied to the tip. Bright and dark lines correspond to conducting tail-totail and insulating head-to-head walls, respectively. (c) - (e) Temperature-dependent cAFM scans obtained at a sixfold domain-wall meeting point. At about $235 \mathrm{~K}$ the conductance contrast at the head-to-head walls vanishes, whereas tail-to-tail walls are resolved down to $160 \mathrm{~K}$. The applied bias voltage in (c), (d) is $3 \mathrm{~V}$ and $10 \mathrm{~V}$ in (e). (f) Integrated conductance data taken at $V_{\text {bias }}=3 \mathrm{~V}$. The exponential fits (solid lines) reflect semiconducting characteristics. The evolution of the optical band gap of $\mathrm{Er}_{0.99} \mathrm{Ca}_{0.01} \mathrm{MnO}_{3}$ as function of temperature is displayed in the inset to (f). The relationship between band gap and temperature follows the Varshni relation for semiconductors (grey dashed line). See SI Note 1 for details and the respective optical measurements. 
sists and subsequent electron beam evaporation of platinum for navigation and for reliably tracking regions of interest in the SPM measurements. The latter are performed on an NTMDT NTEGRA Prima atomic force microscopy (AFM) system (room temperature) and an attocube attoLIQUID2000 AFM (low temperature).
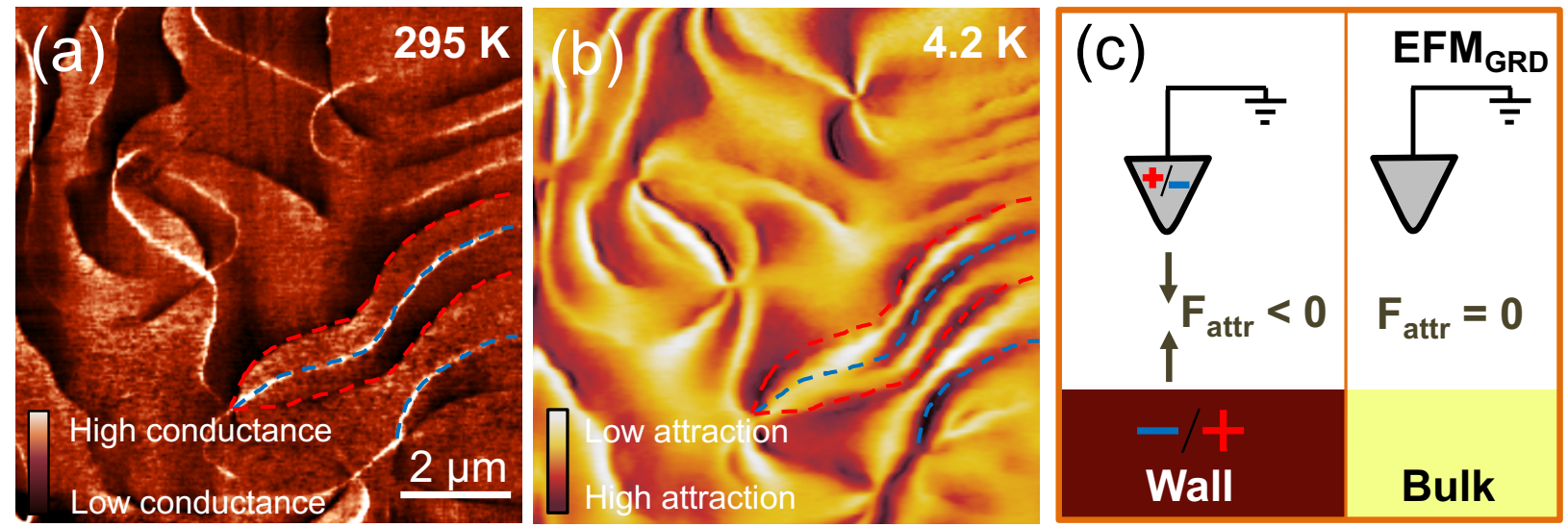

Figure 2: (a) Room-temperature cAFM data obtained on $\mathrm{Er}_{0.99} \mathrm{Ca}_{0.01} \mathrm{MnO}_{3}$ with in-plane polarization as indicated by the white arrows $\left(V_{\text {bias }}=1.5 \mathrm{~V}\right)$. Dashed lines in $(\mathrm{a})$ and $(\mathrm{b})$ indicate the position of head-to-head (red) and tail-to-tail (blue) walls. (b) EFM image at $4.2 \mathrm{~K}$ showing a domain-wall contrast in accordance with (a). (c) Schematic illustration of the setup used for probing local variations in the electrostatic potential. A platinum-coated, grounded tip (GRD) is scanned across the surface with a typical lift height of $30 \mathrm{~nm}$. Uncompensated charges create an image charge in the tip that leads to an additional, charge-induced attractive force $\mathbf{F}_{\text {attr }}$, which is detectable as a change in the phase of the tip oscillation.

Temperature-dependent transport at tail-to-tail and head-to-head walls. We begin with piezoresponse force microscopy (PFM) and conducting atomic force microscopy (cAFM) measurements gained on $\mathrm{Er}_{0.99} \mathrm{Ca}_{0.01} \mathrm{MnO}_{3}$ at room-temperature. The PFM data in Fig. 1a shows a pattern of ferroelectric $180^{\circ}$ domains (in-plane $\mathbf{P}$ ) with meandering domain walls and sixfold meeting points. ${ }^{12,22,25}$ The cAFM scans obtained at the same sample position (Fig. 1b) with a tip voltage $V_{\text {bias }}=3 \mathrm{~V}$ show conducting tail-to-tail (bright) and insulating head-to-head walls (dark), confirming that our $\mathrm{Er}_{0.99} \mathrm{Ca}_{0.01} \mathrm{MnO}_{3}$ sample develops the peculiar domain and domain-wall properties common to hexagonal manganites. ${ }^{3,10}$ Figures 1c-e display selected cAFM scans recorded as function of temperature around a sixfold domain-wall meeting point. Analogous to Fig. 1b, a pronounced conductance contrast is 
observed at both tail-to-tail and head-to-head walls at $295 \mathrm{~K}$ (Fig. 1c). With decreasing temperature, the conductance contrast decreases along with the bulk conductance. Around $235 \mathrm{~K}$ the $\mathrm{cAFM}$ signal at the head-to-head walls reaches the noise level of our setup $(\approx 1 \mathrm{pA})$, whereas the tail-to-tail walls are still visible (Fig. $1 \mathrm{~d}$ ). By increasing $V_{\text {bias }}$, that is, driving a higher current through the sample, the temperature range in which the walls are detectable can be expanded. For $V_{\text {bias }}=10 \mathrm{~V}$, which is the highest bias we apply in our experiments, it is possible to resolve the tail-to-tail walls down to $\approx 160 \mathrm{~K}$ (Fig. 1e).

An overview of the temperature-dependent cAFM measurements is presented in Fig. $1 \mathrm{f}$. Data points are extracted from cAFM maps as in Fig. 1c-e, and bulk and domain-wall values correspond to the mean value averaged over areas of $2 \times 2 \mu \mathrm{m}^{2}$ and $10 \times 30 \mathrm{~nm}^{2}$, respectively. Figure 1f shows that the bulk conductance decreases exponentially with decreasing temperature as expected for a semiconductor. The evolution of the corresponding band gap is displayed in the inset of Fig. 1f, derived by optical transmission spectroscopy as explained in SI Note 1. It is important to note that cAFM records both variations in conductivity as well as changes in the Schottky-barrier formed at the tip-sample interface (SI Note 1), and either contribution can yield exponential scaling as function of temperature. In this work, however, we investigate emergent electrostatics so that we do not pursue this convolution of conduction contributions further.

Most importantly, Fig. 1 shows that semiconducting behavior - with conductance dropping exponentially as the temperature decreases — is measured in both domain and domain wall regions. The latter is intriguing as it potentially leads to the emergence of partially unscreened domain walls at low temperatures: Upon cooling, the increase in bulk polarization due to the pyroelectric effect yields additional bound charges at the walls. The screening of such additional bound charges, however, is substantially hindered by the reduced conductivity at low temperatures.

Electrostatic response at charged domain walls. In order to investigate the domainwall screening at cryogenic temperatures, we perform low-temperature electrostatic force 

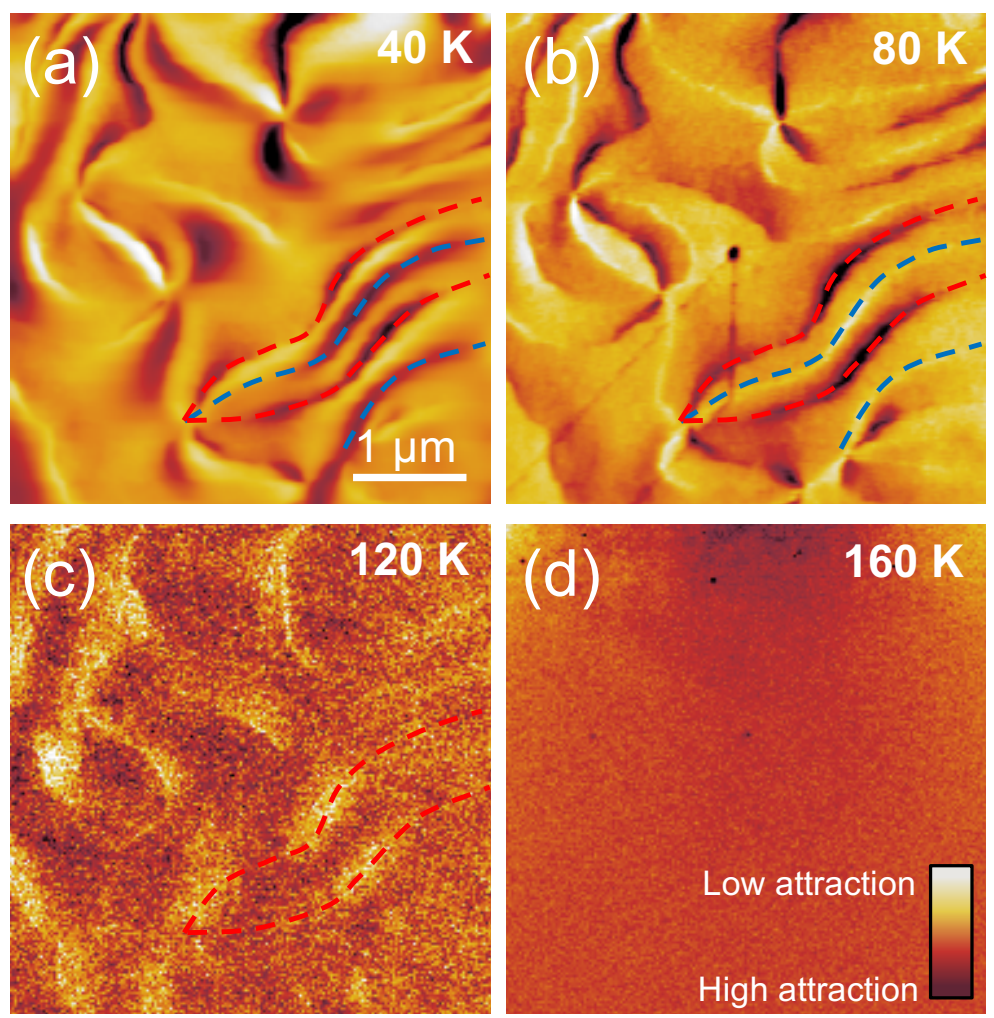

Figure 3: (a) - (d) Temperature-dependent EFM images, showing the evolution of the measured electrostatic potential between $160 \mathrm{~K}$ and $40 \mathrm{~K}$. Phase shifts induced by the local electrostatic field are visible as bright (low attractive force) and dark (high attractive force) regions. Dashed lines indicate the position of head-to-head (red) and tail-to-tail (blue) walls. 
microscopy $(\mathrm{EFM})$ measurements. Figure 2a presents a cAFM image $(T=295 \mathrm{~K})$ showing the distribution of conducting and insulating domain walls similar to Fig. 1b. To record EFM data, an oscillating platinum-coated tip (grounded) is scanned across the region seen in Fig. 2a with a lift height of about $30 \mathrm{~nm}$ (see Fig. 2c). In this setup, both negative and positive charges on the sample surface and in surface-near regions induce a mirror charge on the grounded tip, leading to a charge-induced attractive force $\mathbf{F}_{\text {attr }}$ between tip and sample. $F_{\text {attr }}$ can be detected via a phase shift in the tip oscillation, ${ }^{26,27}$ scaling with the out-of-plane force gradient. ${ }^{28}$

EFM images obtained after cooling the sample to $T=4.2 \mathrm{~K}$ at $\approx 2.5 \mathrm{Kmin}^{-1}$ reveal distinct line-like features as shown in Fig. $2 \mathrm{~b}$ (see SI Note 2 for a topography image). These features coincide with the domain walls in the cAFM image taken at room-temperature (Fig. 2a) as indicated by the dashed lines, corroborating that the domain pattern remains stable down to $4.2 \mathrm{~K} .{ }^{29,30}$ Figure $2 \mathrm{~b}$ shows that away from the sixfold meeting points both tail-to-tail and head-to-head domain walls are darker than the adjacent domains revealing a locally enhanced electrostatic force between the tip and the surface (Fig. 2c).

Upon heating, the EFM wall contrasts decrease as presented in Fig. 3a,b. On a closer inspection, we also find that tail-to-tail and head-to-head walls exhibit different temperature dependencies in EFM. At about $80 \mathrm{~K}$, the contrast at tail-to-tail walls (blue dashed line) first inverts (dark $\rightarrow$ bright) and then vanishes; a similar behavior is observed at head-tohead walls (red dashed line), which are resolved up to higher temperature, i.e., $\approx 120 \mathrm{~K}$ (Fig. 3b-c). At $160 \mathrm{~K}$ the walls are no longer resolved and we detect a homogeneous potential.

In summary the EFM response at head-to-head and tail-to-tail walls vanishes at different temperatures. The distinct temperature-dependencies demonstrate a qualitative difference in the electrostatic fields associated with the two types of domain wall.

Electrostatic far fields at head-to-head and tail-to-tail walls. The measured EFM domain-wall signals can be rationalized by combining analytical and numerical calculations in which $\mathrm{Er}_{0.99} \mathrm{Ca}_{0.01} \mathrm{MnO}_{3}$ is considered as a $p$-type semiconductor similar to previous 
(b)
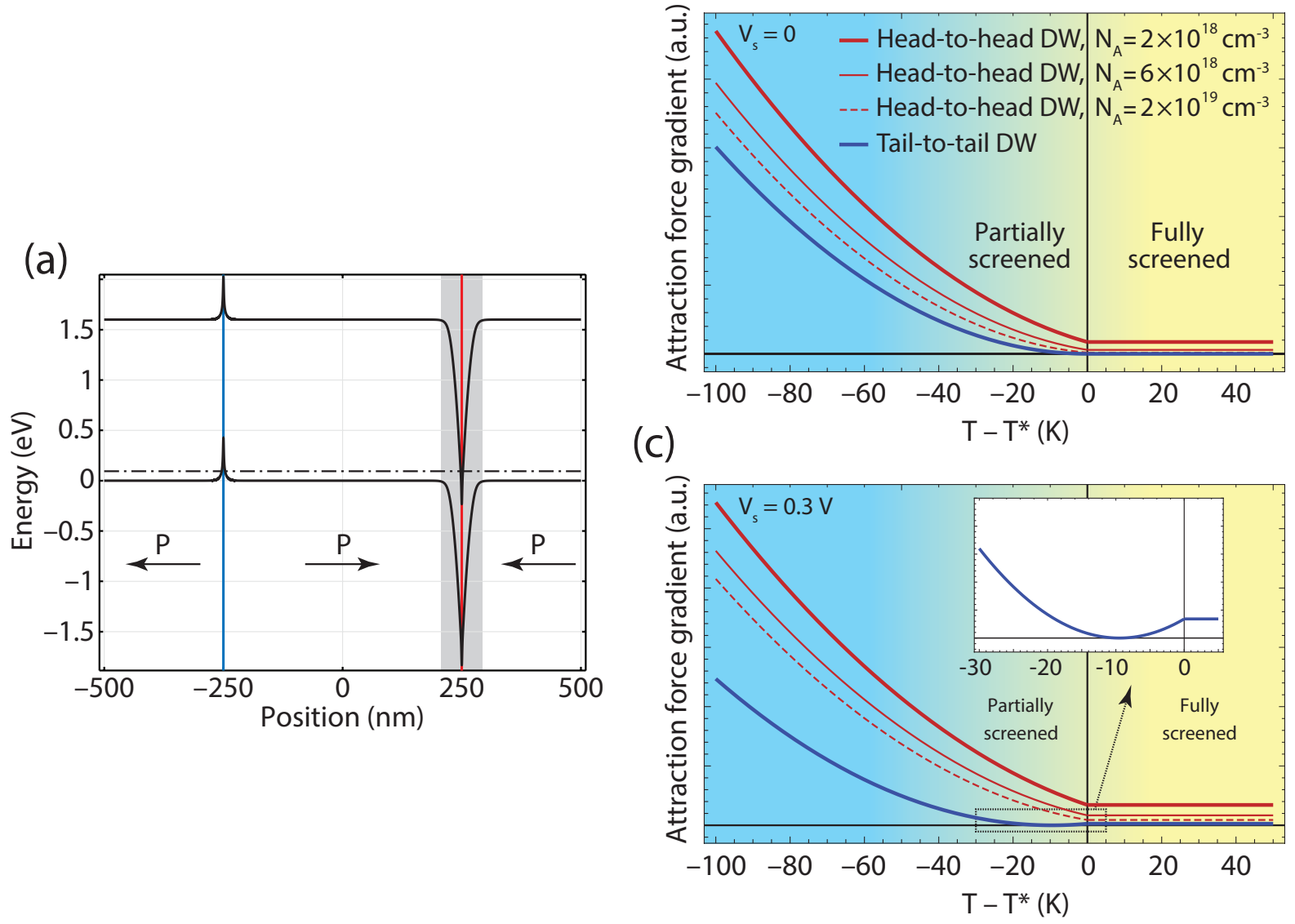

Figure 4: (a) Calculated band structure at charged domain walls in $p$-type hexagonal manganites with band gap $E_{g}=1.6 \mathrm{eV}$ and acceptor density $N_{A}=2 \times 10^{18} \mathrm{~cm}^{-3}$. The black dashed line indicates the Fermi energy, and blue and reds lines indicate the positions of tail-to-tail and head-to-head walls, respectively (see SI Note 3 for details). The depletion layer forming around head-to-head walls is marked in grey. (b) Temperature-dependent evolution of the calculated force gradient of the attractive force acting on the grounded tip at head-to-head and tail-to-tail walls. Lower doping levels result in less effective screening of head-to-head walls by the hole depletion layer and, hence, a stronger EFM response. Blue and yellow backgrounds illustrate temperature regimes with partially and fully screened walls, respectively. (c) Same as (b), but calculated with an additional DC voltage $\left(V_{S}=\right.$ $0.3 \mathrm{~V}$ ) applied to the tip. $V_{S}$ simulates a non-zero surface potential, which reproduces the experimentally observed contrast inversion as shown in the inset to (c) (see SI Note 3 for details). 
studies. $2,15,31$ The calculated band diagram in Fig. 4a is in good agreement with previous analytical $^{2}$ and DFT-based ${ }^{15}$ investigations. The negative bound charge of the tail-to-tail walls is screened by the accumulation of mobile holes within a few unit cells. In contrast, screening of the positive bound charge at head-to-head walls is achieved by means of a hole depletion layer and an electronic inversion layer. ${ }^{15}$

We note that in the considered doping regime, the width of the depletion layer at headto-head walls does not depend on the domain-wall charge, i.e., the spontaneous polarization of the domains, and is given by $w=\sqrt{2 \epsilon E_{g} /\left(e^{2} N_{A}\right)}$ with $\epsilon=13 \epsilon_{0}$ in our system. ${ }^{32}$ For the values of acceptor density used in the calculations, that is, $2 \times 10^{18}$ to $2 \times 10^{19} \mathrm{~cm}^{-3}$, the depletion layer screens about $15 \%$ to $50 \%$ of the maximum bound charge $2 P_{s}$ of the headto-head walls. The rest of the charge is screened by the electronic inversion layer within a few unit cells. ${ }^{15}$

According to Refs. 33,34, the spontaneous polarization of the domains increases as the temperature decreases at a rate of $\sim 1.5 \mathrm{nCcm}^{-2} / \mathrm{K}$, leading to an increase in the domainwall charge. ${ }^{22,34}$ The extra electric fields measured in Figs. 2 and 3 are likely due to the inability of the system to screen the respective extra pyroeletric charge resulting from the exponentially growing relaxation time of the mobile carriers $\tau=\epsilon /\left(e n_{h} \mu\right) \propto \exp \left[E_{F} /\left(k_{B} T\right)\right]$. Here $\mu$ is the hole mobility, $n_{h}$ is the density of mobile holes in the bulk, and $E_{F}$ is the Fermi energy. In particular, as $\tau$ exceeds the data acquisition time of our experiment, electrostatic equilibrium cannot be reached and the extra domain-wall charge remains uncompensated. The latter happens at a characteristic temperature $T^{*}$, which we estimate as $T^{*} \approx 60 \mathrm{~K}$ for the aforementioned parameters and the typical acquisition time $\sim 10^{4} \mathrm{~s}$ of our EFM experiments. This value is in reasonable agreement with the temperature, $\approx 120 \mathrm{~K}$, below which the additional contribution to the electric far fields is detectable (Fig. 3c, signal at the head-to-head walls).

The attractive force on the EFM tip resulting from the charge distribution across the domain walls is shown in Fig. 4b. At $T>T^{*}$ the material is in electrostatic equilibrium (all 
charged domain walls are fully screened) and the band diagram in Fig. 4a determines the charge distribution. In this regime, only head-to-head walls are able to generate a weak and temperature-independent attractive force, which originates from the quadrupolar-like charge distribution associated with the hole depletion layer and the electronic inversion layer. As we do not resolve such a temperature-independent attractive force in our EFM data (Fig. 3), we conclude that this contribution is below the detection limit of our experiment.

However, for $T<T^{*}$, uncompensated pyroelectric charges appear at both head-to-head and tail-to-tail walls with a surface density $\sigma= \pm 2 P_{s} p\left(T^{*}-T\right)$, where $p$ is the pyroelectric constant, leading to a pronouced attractive force between the tip and sample surface at both wall types. These pyroelectric charges provide an additional contribution that adds to the aforementioned weak attractive force at the head-to-head walls, which leads to a stronger EFM response compared to the tail-to-tail walls. The difference in signal strength is in agreement with our experimental observations, explaining why the head-to-head walls are detected at higher temperature than tail-to-tail walls in sensitivity-limited EFM measurements (Fig. 3).

We note that the presence of impurities and crystallographic defects in the sample can increase the relaxation time $\tau$ and hence give rise to a higher $T^{*}$. Furthermore, the EFM contrast inversion observed at the walls can be modeled by including a nonzero surface potential. As we show in Fig. 4(c), the contrast inversion observed at head-to-head walls is reproduced by assuming an additional surface potential, ${ }^{35}$ which we model by a finite voltage $V_{s}$ between probe tip and sample surface.

Conclusions Our work establishes an extraordinary robustness of charged domain walls in hexagonal manganites, which is crucial for domain-wall engineering ${ }^{36,37}$ and to develop them into durable electronic components for domain-wall nanoelectronics. Using low-temperature EFM, we demonstrate the emergence of uncompensated bound domain-wall charges in $\mathrm{Er}_{0.99} \mathrm{Ca}_{0.01} \mathrm{MnO}_{3}$. In contrast to domain walls in proper ferroelectrics, the charged domain walls in this system readily withstand a lack of screening charges, representing a striking 
example for the unique properties of domain walls in improper ferroelectrics.

The exponentially decaying conductance observed at the domain walls in conjunction with previous magnetic force microscopy studies ${ }^{29}$ suggests that domain boundaries in hexagonal manganites act as 2D ferromagnetic semiconductors in their ground state. Such a behavior is intriguing as ferromagnetic semiconductors are capable of providing near-total spin polarization - a concept that has not been addressed in connection with functional (multi-)ferroic domain walls yet, opening up new research opportunities in the field of functional ferroic domain walls.

\section{Funding}

P.S., J.S., M.F. and D.M. acknowledge funding from the SNF (Proposal No. 200021 149192).

D.M. is supported through the NTNU Onsager Fellowship Program and NTNU Outstanding Academic Fellows Program. Z.Y. and E.B. were supported by the US Department of Energy, Office of Science, Basic Energy Sciences, Materials Sciences and Engineering Division under Contract No. DE-AC02-05-CH11231 within the Quantum Materials Program no. KC2202. A.C. and K.S. were supported by the French Government "Investments for the Future" Program, University of Bordeaux Initiative of Excellence (IDEX Bordeaux). M.S. and K.S. acknowledge the funding from the European Research Council (ERC) under the European Union's Horizon 2020 research and innovation program (Grant Agreement No. 724529). M.H. acknowledges funding from ERC (Complexplas), DFG, BW Stiftung, and BMBF.

\section{Acknowledgement}

The authors acknowledge fruitful discussions with E. Soergel and S. V. Kalinin. 


\section{Supporting Information Available}

The Supporting Information is available free of charge on the ACS Publications website at DIO:

Note $1, I(V)$ characteristic and linear transmission spectroscopy measurements; Note 2, topography of the investigated regions; Note 3, numerical modeling for the experimentally found EFM response (PDF)

\section{References}

(1) Hwang, H. Y.; Iwasa, Y.; Kawasaki, M.; Keimer, B.; Nagaosa, N.; Tokura, Y. Emergent phenomena at oxide interfaces. Nature Materials 2012, 11, 103-113.

(2) Eliseev, E. A.; Morozovska, A. N.; Svechnikov, G. S.; Gopalan, V.; Shur, V. Y. Static conductivity of charged domain walls in uniaxial ferroelectric semiconductors. Physical Review B 2011, 83, 1-8.

(3) Meier, D.; Seidel, J.; Cano, A.; Delaney, K.; Kumagai, Y.; Mostovoy, M.; Spaldin, N. A.; Ramesh, R.; Fiebig, M. Anisotropic conductance at improper ferroelectric domain walls. Nature Materials 2012, 11, 284-288.

(4) Sluka, T.; Tagantsev, A. K.; Bednyakov, P.; Setter, N. Free-electron gas at charged domain walls in insulating $\mathrm{BaTiO}_{3}$. Nature Communications 2013, 4, 1808.

(5) Bednyakov, P. S.; Sluka, T.; Tagantsev, A. K.; Damjanovic, D.; Setter, N. Formation of charged ferroelectric domain walls with controlled periodicity. Scientific Reports $\mathbf{2 0 1 5}$ 5,15819 .

(6) Catalan, G.; Seidel, J.; Ramesh, R.; Scott, J. F. Domain wall nanoelectronics. Reviews of Modern Physics 2012, 84 . 
(7) Meier, D. Functional domain walls in multiferroics. Journal of Physics: Condensed Matter 2015, 27, 463003.

(8) Turner, P. W.; McConville, J. P. V.; McCartan, S. J.; Campbell, M. H.; Schaab, J.; McQuaid, R. G. P.; Kumar, A.; Gregg, J. M. Large Carrier Mobilities in ErMnO 3 Conducting Domain Walls Revealed by Quantitative Hall-Effect Measurements. Nano Letters 2018, 10, 6381-6386.

(9) Seidel, J. et al. Conduction at domain walls in oxide multiferroics. Nature Materials 2009, 8, 229-234.

(10) Wu, W.; Horibe, Y.; Lee, N.; Cheong, S.-W.; Guest, J. R. Conduction of Topologically Protected Charged Ferroelectric Domain Walls. Physical Review Letters 2012, 108, 077203.

(11) Schröder, M.; Haußmann, A.; Thiessen, A.; Soergel, E.; Woike, T.; Eng, L. M. Conducting Domain Walls in Lithium Niobate Single Crystals. Advanced Functional Materials 2012, 22, 3936-3944.

(12) Choi, T.; Horibe, Y.; Yi, H. T.; Choi, Y. J.; Wu, W.; Cheong, S.-W. Insulating interlocked ferroelectric and structural antiphase domain walls in multiferroic $\mathrm{YMnO}_{3}$. Nature Materials 2010, 9, 253-258.

(13) Tselev, A.; Yu, P.; Cao, Y.; Dedon, L. R.; Martin, L. W.; Kalinin, S. V.; Maksymovych, P. Microwave a.c. conductivity of domain walls in ferroelectric thin films. Nature Communications 2016, 7, 11630.

(14) Schaab, J.; Skjærvø, S. H.; Krohns, S.; Dai, X.; Holtz, M. E.; Cano, A.; Lilienblum, M.; Yan, Z.; Bourret, E.; Muller, D. A.; Fiebig, M.; Selbach, S. M.; Meier, D. Electrical half-wave rectification at ferroelectric domain walls. Nature Nanotechnology 2018, 13, $1028-1034$. 
(15) Mundy, J. A. et al. Functional electronic inversion layers at ferroelectric domain walls. Nature Materials 2017, 16, 622-627.

(16) Crassous, A.; Sluka, T.; Tagantsev, A. K.; Setter, N. Polarization charge as a reconfigurable quasi-dopant in ferroelectric thin films. Nature Nanotechnology 2015, 10, $614-618$.

(17) Levanyuk, A. P.; Sannikov, D. G. Improper ferroelectrics. Soviet Physics Uspekhi 1974, $17,199-214$.

(18) Tagantsev, A. K.; Cross, L. E.; Fousek, J. Domains in Ferroic Crystals and Thin Films; Springer-Verlag: New York, NY, 2010.

(19) Van Aken, B. B.; Palstra, T. T. M.; Filippetti, A.; Spaldin, N. A. The origin of ferroelectricity in magnetoelectric $\mathrm{YMnO}_{3}$. Nature Materials 2004, 3, 164-170.

(20) Chae, S. C.; Lee, N.; Horibe, Y.; Tanimura, M.; Mori, S.; Gao, B.; Carr, S.; Cheong, S. W. Direct observation of the proliferation of ferroelectric loop domains and vortex-antivortex pairs. Physical Review Letters 2012, 108, 1-5.

(21) Kumagai, Y.; Spaldin, N. A. Structural domain walls in polar hexagonal manganites. Nature Communications 2013, 4, 1540.

(22) Holtz, M. E.; Shapovalov, K.; Mundy, J. A.; Chang, C. S.; Yan, Z.; Bourret, E.; Muller, D. A.; Meier, D.; Cano, A. Topological Defects in Hexagonal Manganites: Inner Structure and Emergent Electrostatics. Nano Letters 2017, 17, 5883-5890.

(23) Schaab, J.; Cano, A.; Lilienblum, M.; Yan, Z.; Bourret, E.; Ramesh, R.; Fiebig, M.; Meier, D. Optimization of Electronic Domain-Wall Properties by Aliovalent Cation Substitution. Advanced Electronic Materials 2016, 2. 
(24) Yan, Z.; Meier, D.; Schaab, J.; Ramesh, R.; Samulon, E.; Bourret, E. Growth of highquality hexagonal $\mathrm{ErMnO}_{3}$ single crystals by the pressurized floating-zone method. Journal of Crystal Growth 2015, 409, 75-79.

(25) Jungk, T.; Hoffmann, Á.; Fiebig, M.; Soergel, E. Electrostatic topology of ferroelectric domains in $\mathrm{YMnO}_{3}$. Applied Physics Letters 2010, 97, 1-4.

(26) Colchero, J.; Gil, A.; Baró, A. M. Resolution enhancement and improved data interpretation in electrostatic force microscopy. Physical Review B 2001, 64, 245403.

(27) Girard, P. Electrostatic force microscopy: principles and some applications to semiconductors. Nanotechnology 2001, 12, 485-490.

(28) Voigtländer, B. Scanning Probe Microscopy; Springer-Verlag: Berlin Heidelberg, 2015; pp $187-204$.

(29) Geng, Y.; Lee, N.; Choi, Y. J.; Cheong, S. W.; Wu, W. Collective magnetism at multiferroic vortex domain walls. Nano Letters 2012, 12, 6055-6059.

(30) Schaab, J.; Trassin, M.; Scholl, A.; Doran, A.; Yan, Z.; Bourret, E.; Ramesh, R.; Meier, D. Ferroelectric domains in the multiferroic phase of $\mathrm{ErMnO}_{3}$ imaged by lowtemperature photoemission electron microscopy. Journal of Physics: Conference Series 2015, 592, 012120.

(31) Zuo, Y.; Genenko, Y. A.; Xu, B.-X. Charge compensation of head-to-head and tail-totail domain walls in barium titanate and its influence on conductivity. J. Appl. Phys. 2014, 116, 044109.

(32) Stengel, M.; Fennie, C. J.; Ghosez, P. Electrical properties of improper ferroelectrics from first principles. Physical Review B 2012, 86, 094112.

(33) Hur, N.; Jeong, I. K.; Hundley, M. F.; Kim, S. B.; Cheong, S.-W. Giant magnetoelectric 
effect in multiferroic $\mathrm{HoMnO}_{3}$ with a high ferroelectric transition temperature. Physical Review B 2009, 79, 134120.

(34) Artyukhin, S.; Delaney, K. T.; Spaldin, N. A.; Mostovoy, M. Landau theory of topological defects in multiferroic hexagonal manganites. Nature Materials 2014, 13, 42-49.

(35) Kalinin, S. V.; Bonnell, D. A. Local potential and polarization screening on ferroelectric surfaces. Physical Review B 2001, 63, 125411.

(36) Salje, E. K. Multiferroic domain boundaries as active memory devices: Trajectories towards domain boundary engineering. ChemPhysChem 2010, 11, 940-950.

(37) Salje, E. K. H. Robust templates for domain boundary engineering in $\mathrm{ErMnO}_{3}$. New Journal of Physics 2016, 18, 051001. 